\title{
Estructura de la comunidad de macroinvertebrados del arroyo Achiras (Córdoba, Argentina): análisis previo a la construcción de una presa
}

\author{
Cristina Mabel Gualdoni \& Ana M. Oberto
}

Departamento de Ciencias Naturales, Facultad de Ciencias Exactas, Físico-Químicas y Naturales, Universidad Nacional de Río Cuarto, A.P.3, 5804 Río Cuarto, Córdoba, Argentina (cgualdoni@exa.unrc.edu.ar)

\begin{abstract}
Macroinvertebrate community structure of the Achiras stream (Córdoba, Argentina): analysis prior to a dam construction. Regulation of rivers and streams modifies parameters that directly influence on the biota. In Achiras-del Gato endorheic basin has completed the construction of a midsize dam that will alter the limnological characteristics of the system and the structure of benthic communities. This study provides the first taxonomic records of macrobenthos of this lotic system and analyzes the spatial and temporal variations of the community structure in the foothills stretches of Achiras stream. Zoobenthos samples were collected and abiotic variables were recorded in runs and riffles, during a year, in two sites located upstream and downstream of the dam area, prior to the dam was built. For each site, habitat and season we determined the abundance, frequency, taxonomic richness, Shannon and evenness, and the data were analyzed using one-way and three-way ANOVA. Attributes of the reference communities were complemented by the determination of ecological indicators and detectors, and exclusive taxa. In the first site 73 taxa were recorded, Simulium was the most abundant and Leptohyphes eximius Eaton, 1882, was the most frequent. In the second site we found 75 taxa, Caenis was the most abundant and Cricotopus sp. 3, was the most frequent. In this study composition and distribution of the biota and indicators taxa are discussed. We determined that the community attributes were affected by the hydrological periods in wich the samples were taken. Achiras stream has a phreatic-pluvial hydrological regime with low flows in winter and high flows in summer Therefore, we expect changes in native biota and in system integrity in response to hydraulic variability reduction when Achiras dam star functioning and alters the natural flow.
\end{abstract}

KEYWORDS. Streams, benthic community structure, spatial and temporal distribution, indicative taxa.

RESUMEN. La regulación de ríos y arroyos mediante diques modifica parámetros que influyen directamente sobre la biota. En la cuenca endorreica Achiras-del Gato ha finalizado recientemente la construcción de una presa de mediana envergadura que alterará las características limnológicas del sistema y la estructura de las comunidades bentónicas. Este estudio aporta los primeros registros del macrobentos de este sistema lótico y analiza la variación espacio-temporal de la estructura de la comunidad en tramos pedemontanos del arroyo Achiras. Durante un año, antes de la construcción de la presa, se colectaron muestras de zoobentos y se registraron variables abióticas en correderas y rabiones de dos sitios del arroyo, uno situado río arriba del área del dique y otro aguas abajo de la misma. Para cada sitio, hábitat y estación del año se determinó abundancia, frecuencia, riqueza taxonómica, Índice de Shannon y equidad, y se analizaron mediante ANOVA de una y tres vías. Los atributos de las comunidades de referencia se complementaron con la determinación de taxa exclusivos e indicadores y detectores ecológicos. En el primer sitio se registraron 73 taxa, Simulium fue más abundante y Leptohyphes eximius Eaton, 1882, más frecuente. En el segundo se hallaron 75 taxa, Caenis fue más abundante y Cricotopus sp. 3 más frecuente. La abundancia y la riqueza fueron significativamente mayores en primavera, y la diversidad en rabiones de invierno. Se determinó que los atributos de la comunidad estuvieron afectados por la época de muestreo. El arroyo Achiras posee un régimen hídrico freático-pluvial, con caudales bajos en invierno y altos en verano, por lo que se esperan cambios en la biota nativa y la integridad del sistema en respuesta a la reducción de la variabilidad hidráulica cuando el funcionamiento de la presa altere el caudal natural.

PALABRAS-CLAVE. Arroyos, estructura de la comunidad bentónica, distribución espacial y temporal, taxa indicadores.

Con excepción de las regiones más inaccesibles del planeta, los sistemas de agua dulce están expuestos a múltiples presiones de origen antrópico: extracción de agua, recepción de efluentes industriales y domésticos, propagación de especies invasoras, alteración de su hidrología, degradación del hábitat, y sobreexplotación de los recursos (AlLAN \& CASTILLO, 2007). La regulación de ríos y arroyos mediante presas se suma a las presiones que desde hace décadas afectan los ecosistemas fluviales. Las presas alteran el ambiente al modificar parámetros que influyen directamente sobre las comunidades de macroinvertebrados bentónicos, peces, algas y vegetación ribereña (PofF \& HART, 2002; Armitage, 2006). La barrera artificial interrumpe el transporte longitudinal de material orgánico y la espiralización de nutrientes, alterando la estructura de las comunidades a lo largo del continuo. Además, los cambios en el régimen natural de las aguas eliminan el disturbio provocado por las crecientes, lo que resulta en una reestructuración de las comunidades (WARD \& STANFORD, 1983; STANFORD
\& WARD, 2001). Las nuevas condiciones influyen en la abundancia y composición de la biota, su tasa de desarrollo y en los niveles de productividad; excluyen los taxa intolerantes a la nueva situación y empobrecen la diversidad. La pérdida de biodiversidad tiene severas implicaciones, como la disminución de la resiliencia y la resistencia a los disturbios, la simplificación del sistema y la pérdida de integridad ecológica (VINSON \& HawKIns, 1998).

En muchas regiones del mundo los perjuicios resultantes de intervenciones antrópicas se incrementan si se tiene en cuenta que la biota de sus ríos y arroyos es aún desconocida. Por este motivo es relevante el estudio de las comunidades de sistemas acuáticos naturales que aun no han sufrido los cambios aparejados al desarrollo y a la urbanización. El manejo integral de los sistemas hídricos requiere de información de base que posibilite generar estudios comparativos pre y post construcción de presas y estimar los cambios ecológicos relacionados con modificaciones de origen humano. La estructura de 
las comunidades acuáticas originales, sus variaciones espacio-temporales, los listados taxonómicos de especies, su biología, sus requerimientos de hábitat y su rango geográfico pasado y presente son conocimientos que constituyen la base para la protección legal de especies amenazadas por acciones antrópicas, y la conservación y recuperación de hábitats (TICKNER et al., 2000; Armitage, 2006; Strayer, 2006).

Una de las principales dificultades al evaluar impactos ecológicos causado por la acción humana sobre los sistemas fluviales, es el desconocimiento de las condiciones bióticas y abióticas imperantes antes de la alteración (MiRAnda et al., 2005). Por lo general, el impacto producido por las presas se estudia comparando secciones fluviales alteradas, ubicadas río abajo de la barrera, con otras localizadas aguas arriba del reservorio (Ogbeibu \& Oribhabor, 2002; Penczak et al., 2006). Sin embargo, cuando los tramos comparados están situados en tierras con distintos usos o existen diferencias geomorfológicas naturales como las de tramos fluviales situados en áreas de piedemonte, puede ser difícil discriminar los cambios atribuidos a la presencia de la presa. En situaciones como estas es importante contar con estudios de las condiciones ambientales y biológicas imperantes antes de la construcción de la presa, para confrontarlas con las registradas luego de la puesta en funcionamiento de la misma.

Las comunidades de invertebrados acuáticos de la provincia de Córdoba, ubicada en el centro de Argentina, han sido estudiadas desde hace más de dos décadas. Se han realizado estudios del macrobentos de ambientes lóticos, su distribución y su ecología (Corigliano et al., 1996; Gualdoni \& Oberto, 1998; Gualdoni \& Corigliano, 2002; Principe et al., 2007, 2010). Sin embargo, en esta región aun permanecen sistemas fluviales cuya biota es desconocida.

En el sur de Córdoba, hasta hace pocos años, se conservaban ríos y arroyos sin intervenciones antrópicas de magnitud, entre ellos el sistema fluvial Achiras-del Gato. En la cuenca alta del sistema, que drena el sur de las Sierras de los Comechingones a través del arroyo Achiras, se ha construido recientemente una presa de mediana envergadura para regulación hídrica. Teniendo en cuenta los antecedentes previos nos proponemos presentar los primeros registros de la diversidad de la fauna macrobentónica a partir de muestras colectadas antes de la construcción de una presa, comparar la distribución espacial y temporal de la estructura de la biocenosis bentónica e identificar las especies indicadoras en dos tramos pedemontanos del arroyo Achiras.

\section{MATERIAL Y MÉTODOS}

El estudio se realizó en el sistema endorreico Achiras-del Gato, ubicado en el sudoeste de la Provincia de Córdoba (Argentina) (Fig. 1). El arroyo Achiras

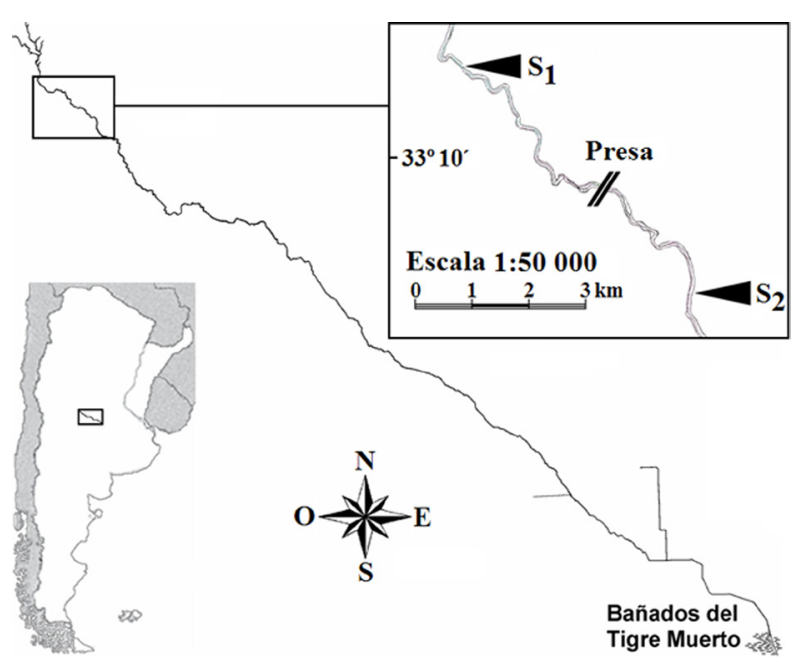

Fig. 1. Localización de los sitios de muestreos estudiados en el arroyo Achiras (Córdoba, Argentina).

nace en la falda sudoriental de las Sierras de los Comechingones y discurre en dirección sur-este hacia la llanura, donde cambia el nombre por arroyo del Gato. Finalmente, luego de recorrer unos $130 \mathrm{~km}$ se derrama en los Bañados del Tigre Muerto. A través de una densa red dendrítica serrana y un único colector en la llanura, el sistema drena una cuenca de aproximadamente 750 $\mathrm{km}^{2}$ (Degiovanni, 2005).

El clima de la región es mesotermal subhúmedohúmedo con alternancia de ciclos húmedos y secos, una temperatura media anual de $16.5^{\circ} \mathrm{C}$ y registros de precipitaciones que, en las últimas décadas (1970 a 2003), promedian los $934.39 \mathrm{~mm}$ anuales, de los cuales el 23\% corresponden al otoño e invierno. Los $77 \%$ restantes se concentran en primavera y verano, estaciones en las que se manifiestan las máximas intensidades de lluvia (Degiovanni, 2005). Otras características climáticas, geológico-geomorfológicas y de ocupación territorial se detallan en el trabajo de Degiovanni (2005).

El arroyo Achiras posee flujo permanente, que depende del aporte freático, con variación de caudal debido a las precipitaciones y picos de creciente violentos pero de corta duración en la época estival. Puesto que las lluvias generalmente comienzan en primavera tardía y se extienden hasta principios de otoño, el caudal presenta dos períodos hidrológicos bien diferenciados, uno de aguas altas y otro de aguas bajas. En la zona de estudio, donde el arroyo es de orden 6 (Degiovanni et al., 2005), la corriente fluye en una sucesión longitudinal de rabiones con turbulencia y substrato de mayor granulometría; correderas poco profundas, con moderada velocidad de corriente y turbulencia superficial escasa o ausente, y pozones más profundos con fondo de arena y limo. Como la mayoría de los arroyos serranos de la región, sus aguas son de buena calidad y están saturadas de oxígeno (Principe et al., 2007). En el área de piedemonte se encuentra emplazada la Presa Achiras, cuya construcción culminó a comienzos de 2008. Esta presa, con una altura de $23 \mathrm{~m}$, controlará las amenazas de eventos catastróficos 
almacenando los excedentes de caudal originados por las precipitaciones en la cuenca alta.

Se seleccionaron dos sitios de muestreo: el sitio $1\left(\mathrm{~S}_{1}\right)\left(33^{\circ} 09^{\prime} 24.44^{\prime} \mathrm{S}, 64^{\circ} 59^{\prime} 04.29^{\prime \prime} \mathrm{W}\right)$ situado a 810 $\mathrm{msnm}$, en un área serrana natural aproximadamente a 1.8 $\mathrm{km}$ río arriba del reservorio, en zona que no se inundará con el agua embalsada, y el sitio $2\left(\mathrm{~S}_{2}\right)\left(33^{\circ} 11^{\prime} 18.36^{\prime \prime} \mathrm{S}\right.$, $64^{\circ} 56^{\prime} 52.70^{\prime \prime} \mathrm{W}$ ), ubicado a $759 \mathrm{msnm}$, en una zona agrícola-ganadera, a $2 \mathrm{~km}$ río abajo de la presa.

Los muestreos se desarrollaron durante un ciclo anual, al comienzo de cada estación del año (otoño, invierno, primavera y verano) entre 2006 y 2007. En el período de aguas altas, cuando las precipitaciones son frecuentes y abundantes, las colectas se realizaron al menos 10 días después de las lluvias. En cada sitio, mediante redes de Surber de $0.09 \mathrm{~m}^{2}$ de área y 300 $\mu \mathrm{m}$ de abertura de malla, se tomaron tres submuestras de zoobentos, en tres hábitats: corredera a la sombra, corredera al sol y rabión, en cada ciclo anual, totalizando 72 submuestras (nueve en cada estación del año, 36 en cada sitio). Las muestras se fijaron con formaldehído al $4 \%$. Paralelamente se registraron las variables hidráulicas del canal: ancho seco y húmedo, profundidad y velocidad de corriente con velocímetro digital Global Flow Probe FP101-FP202; y variables físico-químicas con sensores portátiles: temperatura del aire y del agua, $\mathrm{pH}$, sólidos sedimentables y conductividad. La granulometría del sustrato se registró como porcentaje de cobertura en un marco de 50 x $50 \mathrm{~cm}$, grillado cada $10 \mathrm{~cm}$ y se clasificó según Hynes (1970). Además, se tomaron muestras de agua para análisis químicos en laboratorio: sólidos disueltos totales (SDT), bicarbonatos, sulfatos, cloruros, sodio, potasio, calcio, magnesio, nitratos, fluoruros, dureza total y alcalinidad. Los sólidos sedimentables se determinaron mediante cono de Imhoff.

En el laboratorio los organismos se separaron bajo estéreomicroscopio y microscopio binocular, se determinaron con la máxima resolución taxonómica posible, mediante claves (FernÁNDEZ \& Domínguez, 2001; Domínguez \& FernándeZ, 2009) y se conservaron en alcohol de $70 \%$. Para cada sitio se determinó la abundancia relativa de cada taxa, como porcentaje de abundancia del mismo respecto de la densidad total de organismos en ese sitio, y la frecuencia relativa como porcentaje de presencia de cada taxa respecto al total de muestras del sitio. Para cada situación de muestreo (sitios, hábitats y fechas) se calcularon los atributos de la comunidad bentónica: densidad de cada taxa, riqueza taxonómica, índice de Shannon y equidad (MAgurRan, 1988). Estos dos últimos índices se determinaron utilizando el software estadístico BioDiversity Pro (Mc Aleece, 1997). En este estudio se utilizó riqueza taxonómica en lugar de riqueza específica ya que no todas las identificaciones fueron realizadas a nivel de especie.

Las diferencias de densidad, riqueza, Shannon y equidad entre sitios, hábitats y estaciones del año se compararon mediante análisis de varianza (ANOVA) de una vía. Para determinar el efecto de las situaciones de muestreo se analizaron las interacciones mediante ANOVA de tres vías tomando como factores la estación del año (O, I, P y V), el hábitat (corredera a la sombra, corredera al sol y rabión) y el sitio $\left(\mathrm{S}_{1}\right.$ y $\left.\mathrm{S}_{2}\right)$. En caso de registrar diferencias significativas se aplicó el test a posteriori SNK (Student-Newman-Keuls) para realizar las comparaciones pertinentes. Los análisis se realizaron con el programa INFOSTAT Versión 2010 (Di RiENzo et al., 2010). Para cumplir con los supuestos de normalidad $\mathrm{y}$ homogeneidad de varianzas, los datos de diversidad y equidad se transformaron en $Y^{2}$ y los de abundancia en $\log _{10} Y$.

Para completar la caracterización del ensamble bentónico de cada sitio se determinó el Índice de Preferencia Relativa (IPR) (TiCKNER et al., 2000), dividiendo la abundancia de cada taxa en una cierta situación de muestreo (sitios, hábitats y fechas) por su abundancia total registrada en todas las muestras colectadas. Además, para investigar qué taxa son indicadores ecológicos de un sitio particular, se utilizó el Método de Valor Indicador (Indicator Value - IndVal) propuesto por DuFRENE \& LEGENDRE (1997), que integra en un valor porcentual, la frecuencia de ocurrencia (fidelidad) y la abundancia relativa (concentración) de las especies de un hábitat determinado. Los valores indicadores (VI) más elevados corresponden a especies con alta especificidad y fidelidad a un hábitat particular, y son mejores indicadoras porque se detectan más fácilmente. Las especies con VI intermedios son consideradas especies "detectoras" porque brindan información de más de un hábitat $\mathrm{y}$, por lo tanto, pueden indicar la dirección de cambios en la calidad del hábitat y, eventualmente, su nivel de conservación o perturbación (Dufrene \& Legendre, 1997). Los taxa que con VI significativos superiores al 50\% se consideraron "indicadores" y aquellos que presentaron un valor entre $25 \%$ y $49 \%$, "detectores" (Test de Montecarlo, $\mathrm{p}<0,05$ ). $\mathrm{El}$ análisis se realizó mediante el programa PC-Ord for Windows 4.25 (McCune \& MefFord, 1999).

\section{RESULTADOS}

Los valores medios de los parámetros hidráulicos variaron según los sitios y ambientes (Tab. I). Las velocidades de corriente más elevadas se registraron en los rabiones y las mayores profundidades en las correderas. Los sustratos de roca y bloque predominaron en los rabiones, y en las correderas los de granulometría menor, como gravas y arenas. Se registró una variación temporal en la temperatura del agua, la velocidad de corriente, la profundidad y el ancho mojado del cauce, con los mayores valores en verano (Tab. II). En todas las ocasiones de muestreo tanto las temperaturas ambientales como las precipitaciones estuvieron dentro de las medias normales de cada estación del año. Las 
Tab. I. Valores medios ( \pm DS) de las variables físicas e hidráulicas del canal, registradas en corredera al sol $(\mathrm{Cl})$, corredera a la sombra $(\mathrm{Cm})$ y rabión $(\mathrm{Rb})$ de los dos sitios de muestreo del arroyo Achiras, Córdoba, Argentina.

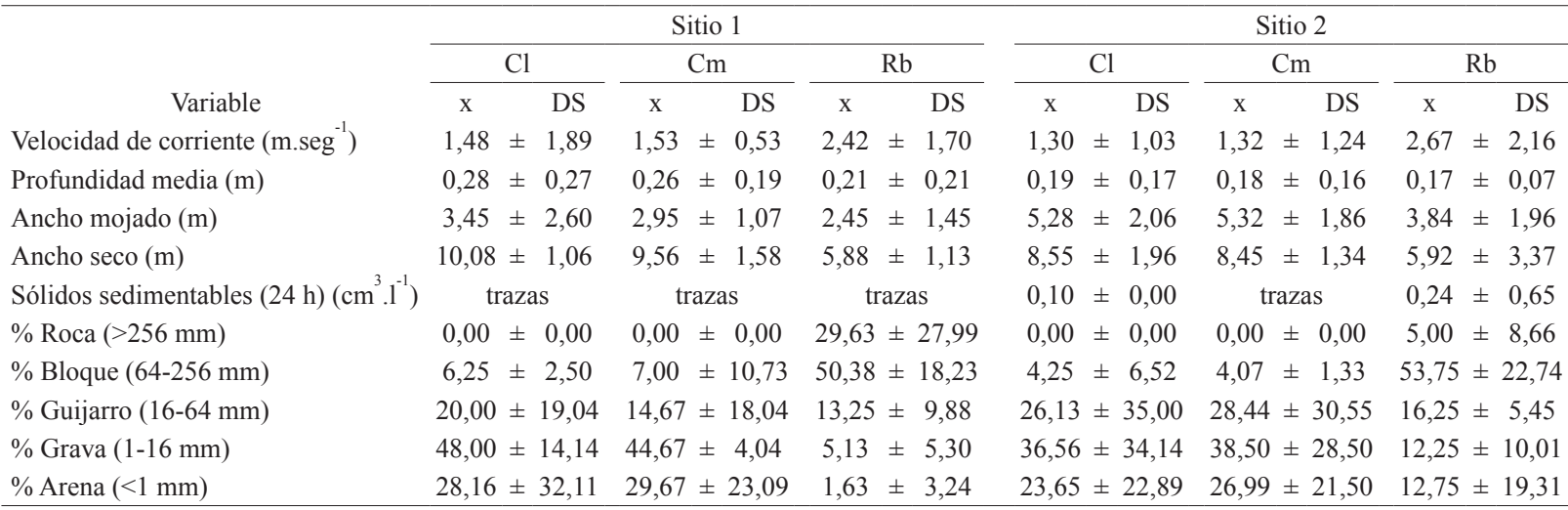

Tab. II. Variación temporal de los registros medios ( \pm DS) de la velocidad de corriente (Vel.), profundidad (Prof.), alveo mojado (A. moj.) y temperatura del agua (Temp.) en los dos sitios de muestreo del arroyo Achiras, Córdoba, Argentina.

\begin{tabular}{|c|c|c|c|c|c|}
\hline & & Vel. $\left(\mathrm{m}^{\mathrm{seg}}{ }^{-1}\right)$ & Prof. (m) & A. moj. (m) & Temp. $\left({ }^{\circ} \mathrm{C}\right)$ \\
\hline \multirow{4}{*}{$\mathrm{S} 1$} & Otoño & $1,98 \pm 2,29$ & $0,29 \pm 0,07$ & $3,62 \pm 0,02$ & $15,00 \pm 1,00$ \\
\hline & Invierno & $0,80 \pm 0,10$ & $0,18 \pm 0,16$ & $1,95 \pm 0,21$ & $13,50 \pm 0,00$ \\
\hline & Primavera & $0,90 \pm 0,08$ & $0,19 \pm 0,04$ & $1,61 \pm 0,40$ & $20,00 \pm 2,00$ \\
\hline & Verano & $3,28 \pm 0,02$ & $0,38 \pm 0,16$ & $4,65 \pm 0,32$ & $23,50 \pm 2,00$ \\
\hline \multirow{4}{*}{$\mathrm{S} 2$} & Otoño & $2,62 \pm 0,11$ & $0,15 \pm 0,04$ & $5,47 \pm 1,27$ & $12,00 \pm 0,00$ \\
\hline & Invierno & $0,51 \pm 0,20$ & $0,11 \pm 0,03$ & $3,46 \pm 0,59$ & $14,00 \pm 0,50$ \\
\hline & Primavera & $0,28 \pm 0,25$ & $0,09 \pm 0,10$ & $2,65 \pm 0,47$ & $20,00 \pm 0,70$ \\
\hline & Verano & $3,70 \pm 1,66$ & $0,33 \pm 0,14$ & $6,69 \pm 0,20$ & $24,50 \pm 1,50$ \\
\hline
\end{tabular}

Tab. III. Variables químicas registradas en las estaciones de verano (V) e invierno (I), en los dos sitios de muestreo del arroyo Achiras, Córdoba, Argentina (SDT, Sólidos Disueltos Totales).

\begin{tabular}{|c|c|c|c|c|}
\hline \multirow[b]{2}{*}{ Variable } & \multicolumn{2}{|c|}{ Sitio 1} & \multicolumn{2}{|c|}{ Sitio 2} \\
\hline & $\mathrm{V}$ & I & $\mathrm{V}$ & $\mathrm{I}$ \\
\hline$\overline{\mathrm{pH}}$ & 7,89 & 7,30 & 7,73 & 7,56 \\
\hline Conductividad $(\mu \mathrm{s} / \mathrm{cm})$ & 214,00 & 202,00 & 230 & 306,00 \\
\hline $\mathrm{SDT}(\mathrm{mg} / \mathrm{l})$ & 185,50 & 179,00 & 219 & 289,00 \\
\hline Bicarbonatos (mg/l) & 102,50 & 110,00 & 145 & 177,50 \\
\hline Sulfato $(\mathrm{mg} / \mathrm{l})$ & 23,60 & 13,40 & 11 & 22,80 \\
\hline Cloruros (mg/l) & 7,15 & 8,60 & 8,6 & 11,40 \\
\hline Sodio (mg/l) & 13,20 & 11,80 & 15,7 & 26,80 \\
\hline Potasio (mg/l) & 2,65 & 2,20 & 5,6 & 4,40 \\
\hline Calcio (mg/l) & 27,60 & 28,00 & 26,4 & 36,80 \\
\hline Magnesio (mg/l) & 6,10 & 4,55 & 5,9 & 7,30 \\
\hline Nitrato (mg/l) & 2,00 & 0,90 & 0 & 1,50 \\
\hline Fluoruro (mg/l) & 0,58 & 0,39 & 0,48 & 0,60 \\
\hline Dureza Total (meq/l) & 1,90 & 1,76 & 1,8 & 2,40 \\
\hline Alcalinidad TAC (meq/l) & 1,65 & 1,80 & 2,3 & 2,80 \\
\hline
\end{tabular}

variables físico-químicas registradas no mostraron alteraciones importantes de la calidad del agua en ninguno de los dos tramos estudiados (Tab. III). Sin embargo, con excepción de los sulfatos y nitratos, todos los valores fueron mayores en $\mathrm{S}_{2}$, entre los que se destacan la conductividad, los SDT y los bicarbonatos.

La fauna de macroinvertebrados bentónicos fue abundante en ambos sitios. En $\mathrm{S}_{1}$ se registró una densidad media de 20123.63 ind. $\mathrm{m}^{-2}$ distribuidos en 73 taxa y en $\mathrm{S}_{2}$ se hallaron 75 taxa que con una densidad promedio de
20673.18 ind. $\mathrm{m}^{-2}$. En ambos tramos Ephemeroptera (con siete especies en ambos sitios) y Diptera (con 28 taxa en $\mathrm{S}_{1}$ y en $\mathrm{S}_{2}$ ) fueron los grupos más abundantes, sin embargo mientras que en el sitio ubicado río arriba predominaron Americabaetis sp., Leptohyphes eximius, Tricorythodes popayanicus, Simulium y Rheotanytarsus sp., en el situado río abajo las mayores densidades correspondieron a Caenis, Dicrotendipes y Rheotanytarsus sp. (Tab. IV). Las especies más frecuentes pertenecieron a Ephemeroptera y Chironomidae, Americabaetis sp., $L$. eximius, T. popayanicus, Thienemannimyia sp. en $\mathrm{S}_{1}$, y Americabaetis sp., Caenis, Thienemannimyia sp. y Cricotopus sp. 3, en $\mathrm{S}_{2}$.

No se detectaron diferencias significativas en la densidad ni en la riqueza taxonómica entre los sitios ni entre los hábitats estudiados (Tab. V, Fig. 2), pero sí entre las estaciones del año $(\mathrm{p}<0,0001)$, con los menores registros medios en verano (4739.22 ind. m $^{-2}$ y 15.83 taxa, respectivamente) y los mayores en primavera (33197.29 ind. $\mathrm{m}^{-2}$ y 29.39 taxa). El índice de Shannon varió significativamente entre los distintos hábitats $y$ entre las fechas de muestreo $(\mathrm{p}=0.0062 ; \mathrm{p}=0.0005$, respectivamente). Los menores valores promedio se hallaron en correderas sol (1.98), en verano (1.93) y en $\mathrm{S}_{1}$ (2.09), los más elevados en rabiones (2.28), en invierno (2.35) y en $\mathrm{S}_{2}$ (2.17). Tampoco se detectaron diferencias significativas en la equidad de los distintos hábitats, aunque esta variable fue significativamente más elevada $(p=0.0064)$ en $S_{2}(0.71)$ que en $S_{1}(0.65)$ y en invierno $(0.72)$ y verano $(0.72)$, que en otoño $(0.62)$ y primavera $(0.65)(\mathrm{p}=0,0025)$. Al evaluar el efecto conjunto de las estaciones del año, los sitios y los hábitats sobre los atributos de la comunidad macrobentónica se determinó que, tanto la riqueza taxonómica como la diversidad y la equidad dependieron de la época, el sitio y el hábitat en que se tomó la muestra. Por su parte, la densidad en cada sitio y en cada hábitat estuvo afectada por la época de muestreo ( $\mathrm{p}<0,0001$ y $\mathrm{p}=0,0016$ respectivamente) (Tab. V).

El IPR calculado para los diferentes taxa en cada situación de muestreo varió de cero a uno. Los IPR más elevados determinan la preferencia del taxon para un 
tramo o situación en particular. En $\mathrm{S}_{1}$ los mayores valores $(\mathrm{IPR}=1)$ correspondieron a 10 taxa, mientras que en $\mathrm{S}_{2}$ a 14 (Tab. VI). Respecto de la preferencia de hábitat, los rabiones presentaron el mayor número de taxa exclusivos y las correderas a la sombra solo fueron preferidas por larvas de Dolichopodidae. Al analizar las muestras según la estación del año se determinaron seis taxa exclusivos de otoño, cinco de invierno, ocho de primavera y solo uno de verano.

Los sitios difirieron también respecto a sus indicadores ecológicos. El Método IndVal permitió determinar 10 taxa indicadores y nueve taxa detectores para $S_{1}$, mientras que para $S_{2}$ resultaron significativos cuatro taxa indicadores y ocho detectores (Test de Montecarlo, $\mathrm{p}<0,05)$ (Tab. VII).

Tab. IV. Lista taxonómica, Abundancia Relativa (A\%) y Frecuencia Relativa ( $\mathrm{F} \%$ ) de macroinvertebrados bentónicos en los dos sitios estudiados del arroyo Achiras, Córdoba, Argentina.

\begin{tabular}{|c|c|c|c|c|}
\hline \multirow[b]{2}{*}{ Taxón } & \multicolumn{2}{|c|}{ Sitio $1(n=36)$} & \multicolumn{2}{|c|}{ Sitio $2(n=36)$} \\
\hline & $\mathrm{A}(\%)$ & $\mathrm{F}(\%)$ & $\mathrm{A}(\%)$ & $\mathrm{F}(\%)$ \\
\hline Hydra sp. & 0,50 & 13,89 & & \\
\hline Dugesiidae indet. & 1,15 & 83,33 & 0,09 & 38,89 \\
\hline Gordioidea indet. & & 5,56 & & 33,64 \\
\hline Nematoda indet. & 0,06 & 27,78 & 0,42 & 38,89 \\
\hline Stenophysa sp. & & & 0,42 & 11,11 \\
\hline Lymnaeidae indet. & & & 0,87 & 52,78 \\
\hline $\begin{array}{l}\text { Gundlachia concentrica } \\
\text { (Orbigny, 1835) }\end{array}$ & & & 0,25 & 38,89 \\
\hline Planorbidae indet. & 0,09 & 66,67 & 0,07 & 22,22 \\
\hline Bivalvia indet. & 0,01 & 5,56 & 0,08 & 22,22 \\
\hline Chaetogaster sp. & 0,02 & 2,78 & 0,04 & 5,56 \\
\hline Amphichaeta sp. & & & 0,04 & 5,56 \\
\hline Homochaeta sp. & & 2,78 & 0,31 & 16,67 \\
\hline Stylaria sp. & & & 0,01 & 2,78 \\
\hline Naididae indet. & 3,27 & 69,44 & 6,45 & 77,78 \\
\hline Tubificidae indet. & & 2,78 & 0,57 & 27,78 \\
\hline Lumbriculidae indet. & 0,02 & 22,22 & 0,10 & 30,56 \\
\hline Hirudiniforme indet. & & & 0,03 & 16,67 \\
\hline Acari indet. & 2,28 & 75,00 & 5,32 & 63,89 \\
\hline Hyalella curvispina Shoemaker, 1942 & & 2,78 & 0,01 & 11,11 \\
\hline Americabaetis sp. & 8,75 & 91,67 & 3,48 & 86,11 \\
\hline Baetodes sp. & & 2,78 & & 2,78 \\
\hline $\begin{array}{l}\text { Camelobaetidius penai } \\
\text { (Traver \& Edmunds, 1968) }\end{array}$ & 2,55 & 52,78 & 0,19 & 44,44 \\
\hline Paracloeodes sp. & 0,04 & 11,11 & 0,05 & 19,44 \\
\hline Caenis sp. & 3,16 & 75,00 & 10,95 & 83,33 \\
\hline $\begin{array}{l}\text { Leptohyphes eximius } \\
\text { Eaton, } 1882\end{array}$ & 10,55 & 97,22 & 2,02 & 75,00 \\
\hline $\begin{array}{l}\text { Tricorythodes popayanicus } \\
\text { Domínguez, } 1982\end{array}$ & 9,89 & 94,44 & 0,32 & 63,89 \\
\hline Aeshnidae indet. & & 2,78 & 0,01 & 2,78 \\
\hline Lestidae indet. & 0,01 & 5,56 & & 5,56 \\
\hline Coenagrionidae indet. & 0,71 & 69,44 & 0,26 & 22,22 \\
\hline Calopterigidae indet. & 0,02 & 5,56 & & \\
\hline Progomphus sp. & 0,01 & 8,33 & 0,15 & 52,78 \\
\hline Libellulidae indet. & & & 0,02 & 13,89 \\
\hline Ambryssus sp. & 0,01 & 11,11 & 0,02 & 5,56 \\
\hline Chimarra sp. & 0,50 & 36,11 & 0,08 & 19,44 \\
\hline Smicridea sp. & 1,01 & 61,11 & 0,21 & 27,78 \\
\hline Oxyethira sp. & 0,06 & 25,00 & 0,07 & 19,44 \\
\hline Metrichia sp. & 0,85 & 72,22 & 0,49 & 38,89 \\
\hline
\end{tabular}

Tab. IV. (Cont.)

\begin{tabular}{|c|c|c|c|c|}
\hline \multirow[b]{2}{*}{ Taxón } & \multicolumn{2}{|c|}{ Sitio $1(n=36)$} & \multicolumn{2}{|c|}{ Sitio $2(n=36)$} \\
\hline & $\mathrm{A}(\%)$ & $\mathrm{F}(\%)$ & $\mathrm{A}(\%)$ & $\mathrm{F}(\%)$ \\
\hline Hydroptila sp. & 0,64 & 50,00 & 0,90 & 38,89 \\
\hline Nectopsyche sp. & 0,01 & 2,78 & 0,02 & 13,89 \\
\hline Marilia sp. & 0,22 & 13,89 & 0,02 & 11,11 \\
\hline Glossosomatidae indet. & & 5,56 & & \\
\hline Mexitrichia sp. & 0,04 & 11,11 & & \\
\hline Protoptila sp. & 0,02 & 16,67 & & \\
\hline Petrophila sp. & 0,05 & 27,78 & 0,40 & 25,00 \\
\hline Berosus patruellis Voss, 1946 & & 2,78 & 0,27 & 41,67 \\
\hline Enochrus (Metydrus) sp. & & & 0,01 & 8,33 \\
\hline Hidrophylidae indet. & 0,01 & 2,78 & & \\
\hline Cylloepus sp. & 0,05 & 41,67 & 0,02 & 22,22 \\
\hline Austrelmis sp. & 0,26 & 80,56 & 0,56 & 75,00 \\
\hline Macrelmis sp. & 0,21 & 77,78 & & 2,78 \\
\hline Heterelmis sp. & 0,44 & 69,44 & 0,02 & 19,44 \\
\hline Microcylloepus sp. & 0,15 & 52,78 & 0,01 & 11,11 \\
\hline Helichus sp. & 0,02 & 13,89 & & 5,56 \\
\hline Tipulidae indet. & 0,02 & 5,56 & 0,03 & 8,33 \\
\hline Maruina sp. & 0,77 & 16,67 & & \\
\hline Ceratopogonidae sp. 1 & 0,01 & 2,78 & & \\
\hline Ceratopogonidae sp. 2 & 0,13 & 25,00 & 0,40 & 30,56 \\
\hline Simulium sp. & 24,86 & 75,00 & 1,53 & 72,22 \\
\hline Polypedilum sp. & 1,17 & 80,56 & 8,92 & 80,56 \\
\hline Cryptotendipes sp. & & & & 5,56 \\
\hline Parachironomus sp. & & & 0,03 & 8,33 \\
\hline Dicrotendipes sp. & 0,02 & 11,11 & 11,22 & 69,44 \\
\hline Cryptochironomus sp. & 0,02 & 11,11 & & \\
\hline Cladotanytarsus sp. & 0,06 & 19,44 & 1,98 & 44,44 \\
\hline Rheotanytarsus sp. & 9,48 & 88,89 & 21,27 & 83,33 \\
\hline Pseudochironomus sp. & & & 0,86 & 41,67 \\
\hline Djalmabatista sp. & 0,56 & 55,56 & 0,13 & 13,89 \\
\hline Larsia $\mathrm{sp}$ & 0,12 & 27,78 & & 5,56 \\
\hline Pentaneura sp. & 0,91 & 36,11 & 0,07 & 22,22 \\
\hline Thienemannimyia sp. & 4,11 & 94,44 & 3,70 & 86,11 \\
\hline Corynoneura sp. & 1,37 & 66,67 & 0,46 & 47,22 \\
\hline Thienemanniella sp. & 0,36 & 50,00 & 0,55 & 25,00 \\
\hline Onconeura sp. & 2,16 & 83,33 & 1,12 & 47,22 \\
\hline Lopescladius sp. & 0,06 & 22,22 & 0,07 & 16,67 \\
\hline Nanocladius sp. & 0,80 & 25,00 & 0,09 & 11,11 \\
\hline Cricotopus sp. 2 & 0,12 & 22,22 & 0,43 & 41,67 \\
\hline Cricotopus sp. 3 & 4,54 & 83,33 & 8,49 & 100,00 \\
\hline Orthocladius sp. & 0,03 & 22,22 & 0,65 & 25,00 \\
\hline Parametriocnemus sp. 1 & 0,34 & 47,22 & 2,21 & 75,00 \\
\hline Parametriocnemus sp. 2 & & & & 2,78 \\
\hline Stratiomyidae indet. & 0,15 & 13,89 & 0,01 & 2,78 \\
\hline Empididae indet. & 0,15 & 36,11 & 0,10 & 19,44 \\
\hline Dolichopodidae indet. & & 2,78 & & \\
\hline Ephydridae indet. & 0,01 & 5,56 & & 2,78 \\
\hline Muscidae indet. & 0,05 & 8,33 & 0,01 & 2,78 \\
\hline
\end{tabular}

\section{DISCUSIÓN}

En los sitios estudiados no se han registrado nuevos taxa respecto de otros sistemas lóticos de la región central de Argentina (CoRIgliano et al., 1996; Gualdoni \& Oberto, 1998; Gualdoni \& Corigliano, 2002; Principe et al., 2007, 2010). Sin embargo, es 

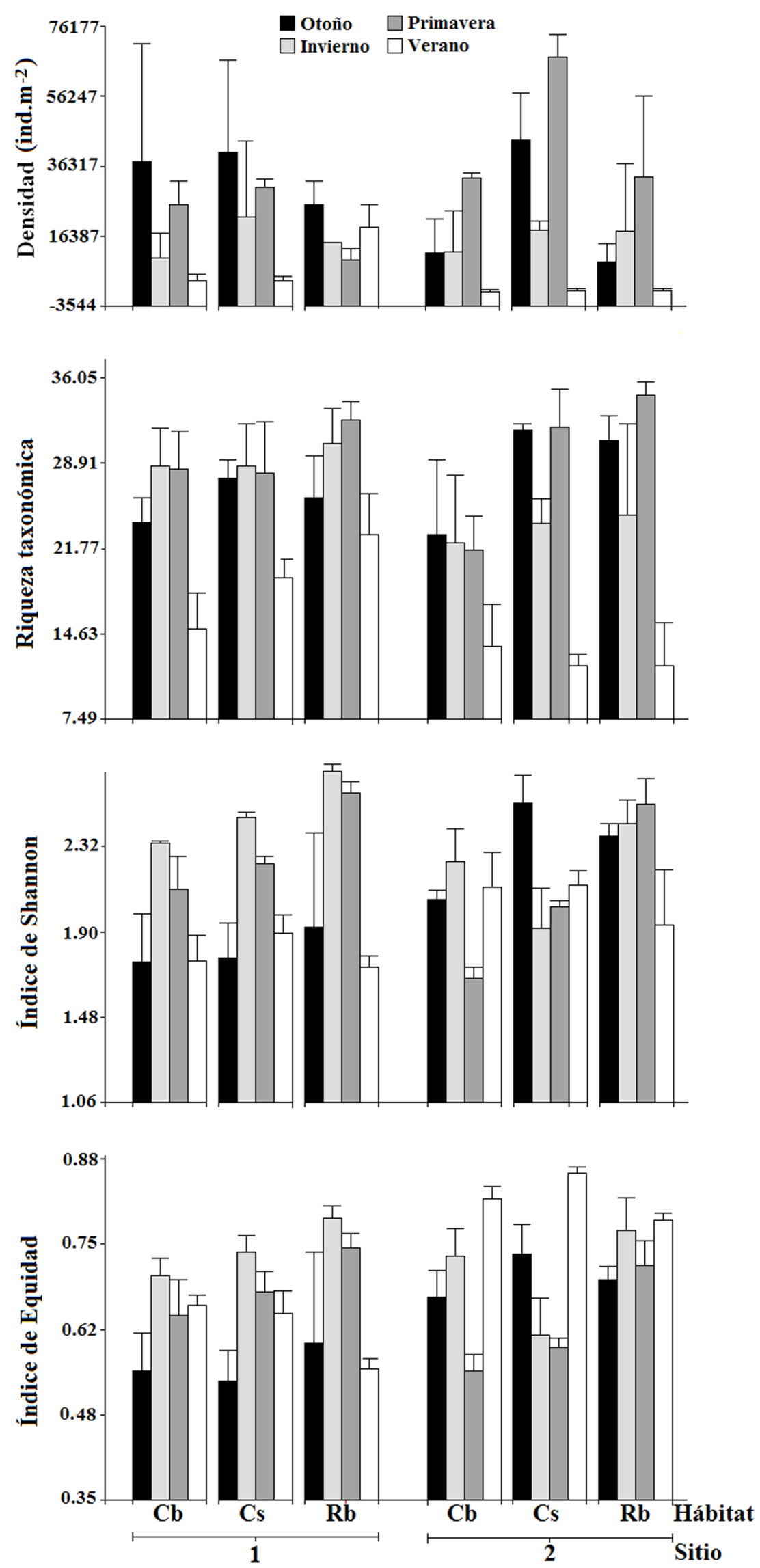

Fig. 2. Variación espacio temporal de densidad, riqueza taxonómica, diversidad y equidad de la comunidad bentónica en correderas a la sombra $(\mathrm{Cb})$, correderas al sol $(\mathrm{Cs})$ y rabiones $(\mathrm{Rb})$ de los dos sitios de muestreo del arroyo Achiras, Córdoba, Argentina. 
Tab. V. Resultado del ANOVA de tres vías que evaluó el efecto de fecha, sitio y hábitat, y los términos de la interacción sobre los atributos de la comunidad bentónica del arroyo Achiras (F, valor F de Fisher; gl, grados de libertad).

\begin{tabular}{|c|c|c|c|}
\hline Fuente de variación & $\mathrm{gl}$ & $\mathrm{F}$ & $\mathrm{p}$ \\
\hline \multicolumn{4}{|l|}{ Densidad } \\
\hline Estación del año & 3 & 75,35 & $<0,0001$ \\
\hline Sitio & 1 & 16,78 & 0,0002 \\
\hline Hábitat & 2 & 5,24 & 0,0087 \\
\hline Estación $x$ sitio & 3 & 21,91 & $<0,0001$ \\
\hline Estación x hábitat & 6 & 4,28 & 0,0016 \\
\hline Sitio $\mathrm{x}$ hábitat & 2 & 2,08 & 0,1363 \\
\hline Estación $\mathrm{x}$ sitio $\mathrm{x}$ hábitat & 6 & 1,2 & 0,3233 \\
\hline \multicolumn{4}{|l|}{ Riqueza Taxonómica } \\
\hline Estación del año & 3 & 53,18 & $<0,0001$ \\
\hline Sitio & 1 & 9,07 & 0,0041 \\
\hline Hábitat & 2 & 11,73 & 0,0001 \\
\hline Estación $\mathrm{x}$ sitio & 3 & 6,86 & 0,0006 \\
\hline Estación $\mathrm{x}$ hábitat & 6 & 1,68 & 0,1467 \\
\hline Sitio $\mathrm{x}$ hábitat & 2 & 0,92 & 0,4066 \\
\hline Estación $\mathrm{x}$ sitio $\mathrm{x}$ hábitat & 6 & 2,31 & 0,0484 \\
\hline \multicolumn{4}{|l|}{ Índice de Shannon } \\
\hline Estación del año & 3 & 24,84 & $<0,0001$ \\
\hline Sitio & 1 & 2,51 & 0,1197 \\
\hline Hábitat & 2 & 22,55 & $<0,0001$ \\
\hline Estación $\mathrm{x}$ sitio & 3 & 29,24 & $<0,0001$ \\
\hline Estación $\mathrm{x}$ hábitat & 6 & 9,33 & $<0,0001$ \\
\hline Sitio x hábitat & 2 & $4,4 \mathrm{E}-3$ & 0,9956 \\
\hline Estación $\mathrm{x}$ sitio $\mathrm{x}$ hábitat & 6 & 2,59 & 0,0298 \\
\hline \multicolumn{4}{|l|}{ Equidad } \\
\hline Estación del año & 3 & 21,62 & $<0,0001$ \\
\hline Sitio & 1 & 34,84 & $<0,0001$ \\
\hline Hábitat & 2 & 7,13 & 0,0019 \\
\hline Estación $\mathrm{x}$ sitio & 3 & 39,52 & $<0,0001$ \\
\hline Estación $\mathrm{x}$ hábitat & 6 & 7,79 & $<0,0001$ \\
\hline Sitio x hábitat & 2 & 0,18 & 0,8365 \\
\hline Estación $\mathrm{x}$ sitio $\mathrm{x}$ hábitat & 6 & 2,56 & 0,0311 \\
\hline
\end{tabular}

probable que aguas abajo del nuevo reservorio la estructura de la biocenosis se modifique cuando la obra comience a funcionar (WARD \& STANFORD, 1983; AlLAN \& CASTILlo, 2007). Los valores de riqueza taxonómica hallados en el arroyo Achiras coinciden con los de arroyos serranos de la región y se encuentran en el rango de estimaciones de riqueza predichas para el hiporritron y los tramos de transición ritron-potamon de zonas templadas (Corigliano et al., 1996). Estudios previos de la comunidad bentónica en otras cuencas de las sierras del sur de Córdoba, han contabilizado entre 69 y 90 taxa (Corigliano et al., 2004; Principe et al., 2007; Gualdoni et al., 2011). Grupos cosmopolitas como Ephemeroptera y las familias de Diptera, Simulidae y Chironomidae, fueron los dominantes del bentos, en coincidencia con lo reportado para otros sistemas locales de semejantes características (GUALDONI \& CoRIGLIANO, 1991; Corigliano et al., 1996; Corigliano et al., 2001). Los dos sitios del arroyo Achiras presentaron sustrato de rocas, bloques y arena, aguas correntosas, limpias y bien oxigenadas, características propicias para el desarrollo de los estados preimaginales de estos insectos (WARD, 1992;
Rosenberg \& Resh, 1993). Además, por su sensibilidad a los contaminantes, tanto los efemerópteros como los simúlidos son considerados indicadores de aguas de buen calidad (Coscarón-Arias, 2009; Fernández \& Domínguez, 2001). Su dominancia confirmaría la buena calidad del agua detectada por las variables químicas registradas, aun cuando en el tramo ubicado río abajo se detectaron mayores valores de conductividad, SDT y bicarbonatos, probablemente asociados a la actividad agrícola-ganadera del entorno.

En los ambientes lóticos, la estructura de la comunidad bentónica resulta de una conjunción de variables ambientales, como velocidad de corriente y granulometría del sustrato (Hynes, 1970; Allan \& CAstillo, 2007). Entre los atributos biológicos evaluados, solo se detectó diferencia significativa entre hábitats para la diversidad. Las estimaciones más elevadas de esta variable se observaron en los rabiones. En estos ambientes también se registró el mayor número de taxa exclusivos, evidenciados por valores de IPR igual a uno. Los rabiones se caracterizan por mayores granulometrías de sedimento y velocidades de corriente, lo cual equivale a mayor heterogeneidad de hábitat (PrinciPe et al., 2007). En los sistemas lóticos los ambientes más heterogéneos y complejos brindan una mayor oferta de hábitats y han sido asociadas a altos valores de diversidad (VINSON \& Hawkins, 1998). Por otra parte, el sustrato de granulometría mediana y grande actúa como barrera reteniendo restos orgánicos, especialmente vegetales, que aportan refugio y alimento a muchos invertebrados e incrementan aún más la heterogeneidad del hábitat al proporcionar más sustrato para que se establezcan los organismos (Sмоске et al., 1989; GJERLøv et al., 2003). Resultados semejantes han sido mencionados para ríos del sur-este de Brasil (BAPTISTA et al., 2001).

Los atributos de la comunidad resultaron afectados por la época del año en que se realizó el muestreo. Para todas las variables biológicas registradas, se detectaron diferencias significativas entre las estaciones del año, con los menores valores densidad, riqueza y diversidad en los muestreos de verano (aguas altas) y los mayores en primavera temprana cuando aun no ha comenzado el período de lluvias. El arroyo Achiras posee un régimen hídrico freático-pluvial, con caudales menores y más estables durante invierno y primavera temprana, cuando las lluvias son escasas y el arroyo depende principalmente del aporte freático. En este período se generan condiciones hidrológicas que favorecen la estabilidad de los biotopos, por lo cual los organismos tienen mayores oportunidades de asociarse con un microhábitat específico y aumentar sus densidades (PofF \& Ward, 1989; Poff et al., 1997; Poff \& Zimmerman, 2010) y riqueza taxonómica (DEATH \& Winterbourn, 1995). Durante el verano y principios de otoño las precipitaciones frecuentes ocasionaron crecientes de corta duración que movilizaron el sustrato. Si bien los 
Tab. VI. Macroinvertebrados bentónicos del arroyo Achiras asociados a cada sitio, hábitat y estación del año. Solo se muestran los taxa con IPR $=1$.

\begin{tabular}{|c|c|c|c|c|}
\hline \multirow[t]{14}{*}{ Sitio } & S1 & S2 & & \\
\hline & Hydra & Lymnaeidae & & \\
\hline & Calopterigidae & Gundlachia concentrica & & \\
\hline & Glossosomatidae & Amphichaeta & & \\
\hline & Mexitrichia & Stylaria & & \\
\hline & Protoptila. & Tubificidae & & \\
\hline & Hidrophylidae & Hirudiniforme & & \\
\hline & Maruina & Libellulidae & & \\
\hline & Ceratopogonidae sp. 1 & Enochrus (Metydrus) & & \\
\hline & Cryptochironomus sp. & Cryptotendipes & & \\
\hline & Dolichopodidae & Parachironomus sp. & & \\
\hline & & Dicrotendipes & & \\
\hline & & Pseudochironomus sp. & & \\
\hline & & Parametriocnemus sp. 2 & & \\
\hline \multirow[t]{6}{*}{ Hábitat } & Corredera sol & Corredera sombra & Rabion & \\
\hline & Amphichaeta & Dolichopodidae & Stylaria & \\
\hline & Calopterigidae & & Baetodes & \\
\hline & Ceratopogonidae sp. 1 & & Glossosomatidae & \\
\hline & & & Cryptotendipes & \\
\hline & & & Parametriocnemus sp. 2 & \\
\hline \multirow[t]{9}{*}{ Estación } & Otoño & Invierno & Primavera & Verano \\
\hline & Chaetogaster & Ceratopogonidae sp. 1 & Stenophysia & Baetodes \\
\hline & Glossosomatidae & Cryptotendipes & Amphichaeta & \\
\hline & Hidrophylidae & Cryptochironomus sp. & Stylaria & \\
\hline & Parachironomus sp. & Dolichopodidae & Calopterigidae & \\
\hline & Parametriocnemus sp. 2 & Ephydridae & Mexitrichia & \\
\hline & & & Enochrus (Metydrus) & \\
\hline & & & Nanocladius sp. & \\
\hline & & & Stratiomyidae & \\
\hline
\end{tabular}

Tab. VII. Taxa indicadores $(\mathrm{VI}>50)$ y detectores $(25<\mathrm{IV} \leq 49)$ para cada sitio del arroyo Achiras (p, valores significativos; VI, Valor Indicador).

\begin{tabular}{|c|c|c|c|c|c|c|}
\hline & \multirow[t]{2}{*}{ Sitio de muestreo } & \multicolumn{2}{|c|}{ S1 } & & \multicolumn{2}{|c|}{ S2 } \\
\hline \multirow{11}{*}{ 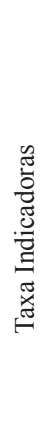 } & & VI & $\mathrm{p}$ & & VI & $\mathrm{p}$ \\
\hline & Tricorythodes sp. & 91,5 & 0,001 & Polypedilum sp. & 71,4 & 0,001 \\
\hline & Leptohyphes sp. & 81,3 & 0,001 & Dicrotendipes sp. & 69,3 & 0,001 \\
\hline & Macrelmis sp. & 77,2 & 0,001 & Parametriocnemus sp.1 & 65,3 & 0,001 \\
\hline & Dugesiidae & 77,1 & 0,001 & Lymnaeidae & 52,8 & 0,001 \\
\hline & Simulium sp. & 70,6 & 0,004 & & & \\
\hline & Heterelmis sp. & 65,8 & 0,001 & & & \\
\hline & Americabaetis sp. & 65,2 & 0,026 & & & \\
\hline & Onconeura sp. & 54,5 & 0,04 & & & \\
\hline & Coenagrionidae & 50,5 & 0,005 & & & \\
\hline & Smicridea $\mathrm{sp}$. & 50,4 & 0,002 & & & \\
\hline \multirow{9}{*}{ 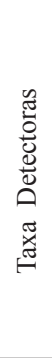 } & Corynoneura sp. & 49,7 & 0,031 & Progomphus sp. & 49,8 & 0,001 \\
\hline & Microcylloepus sp. & 49,6 & 0,001 & Cladotanytarsus sp. & 43,1 & 0,003 \\
\hline & Caenis penai & 49,1 & 0,012 & Berosus patruellis & 41,4 & 0,001 \\
\hline & Metrichia sp. & 45,3 & 0,034 & Pseudochironomus sp. & 41,7 & 0,001 \\
\hline & Djalmabatista sp. & 45,0 & 0,003 & Gundlachia concentrica & 38,9 & 0,001 \\
\hline & Chimarra sp. & 31,1 & 0,033 & Nematoda & 34,1 & 0,034 \\
\hline & Cylloepus sp. & 30,6 & 0,031 & Cricotopus sp. 2 & 32,5 & 0,046 \\
\hline & Larsia sp. & 27,1 & 0,003 & Tubificidae & 27,8 & 0,002 \\
\hline & Nanocladius sp. & 22,5 & 0,035 & & & \\
\hline
\end{tabular}


muestreos nunca se realizaron inmediatamente después de las lluvias, es probable que las disminuciones en los registros del bentos pueda ser atribuido a la acción de las derivas catastróficas que desprenden y desplazan la fauna bentónica aguas abajo, disminuyendo su abundancia local (LANCASTER, 2008). Por otra parte, PofF \& WARD (1991) consideran que cuando las condiciones hidrológicas permanecen estables durante un período prolongado, los organismos tienen mayores oportunidades de asociarse con un hábitat específico y aumentar sus densidades, mientras que en períodos hidrológicamente más variables las condiciones del hábitat son menos estables y los organismos están mas expuestos a las posibilidades de derivar. Estudios previos de deriva bentónica, realizados en la cuenca del río Chocancharava también han puesto en evidencia que entre los principales factores que afectan la estructura de la comunidad bentónica están el aumento de caudal y la inestabilidad del sustrato (GuALDONI \& Corigliano, 2002).

El establecimiento de una presa trae aparejado cambios del ecosistema fluvial, cuyos efectos se evidencian en el entorno inmediato y en el continuo fluvial. Una de las alteraciones más importantes es la modificación del régimen de caudal natural, cuyas variaciones temporales generan las diferencias ecológicas y evolutivas dentro y entre los ríos (PofF et al., 2007). Las presas actúan homogeneizando el caudal (por ejemplo: disminuyen la frecuencia y duración de las inundaciones o los picos de crecientes o cambian la estacionalidad de los mismos). Estos fenómenos pueden inducir a una variedad de respuestas como reducción de la abundancia y riqueza de especies, e incluso la pérdida de especies endémicas y sensibles, y el aumento en la densidad de especies exóticas (Poff \& Zimmerman, 2010). Este estudio puso de manifiesto la relación entre los atributos de la comunidad bentónica y las variaciones de caudal, por lo tanto son de esperar cambios en la biota nativa y la integridad del ecosistema como respuesta a la reducción de la variabilidad hidráulica cuando el funcionamiento de la presa altere el caudal natural.

No se detectaron diferencias significativas entre sitios para la mayoría de las variables biológicas consideradas, no obstante el $\mathrm{S}_{2}$ resulto más diverso y con mayor equidad que el $\mathrm{S}_{1}$, lo cual era de esperar, ya que tanto el paisaje como el uso de la tierra en cada uno son diferentes. En el $\mathrm{S}_{1}$, ubicado en un área natural de sierras bajas, la única actividad antrópica es la recreación en época estival. En este tramo el ensamble bentónico estuvo caracterizado por un menor número de taxa exclusivos y diez especies indicadoras estadísticamente significativas, entre las cuales $T$. popayanicus y $L$. eximius fueron las que caracterizaron mejor el ambiente. Estas especies son comunes en arroyos no contaminados, con buena corriente y fondos pedregosos y gravosos del centro de Argentina (Molineri, 2003, 2010). El segundo sitio se localiza en un tramo que discurre en un área de uso agrícola-ganadero extensivo. Entre los taxa exclusivos de este tramo sobresalieron los moluscos (Gastropoda y Bivalvia), anélidos (Naididae, Tubificidae e Hirudiniforme) y algunas especies de Chironomidae. Por su mayor especificidad a las condiciones del hábitat los mayores VI correspondieron a Lymnaeidae y tres taxa de quironómidos, entre ellas Dicrotendipes, que fue también la más abundante del tramo. Tanto los taxa exclusivos como los indicadores y detectores de este sitio son grupos que están asociados a corrientes más suaves y sedimentos con predominio de arena fina (MARCHESE, 2009). La mayoría de los quironómidos hallados en ambos sitios son géneros con amplios límites de tolerancia, por lo cual es difícil relacionarlos con alguna condición en particular (PAGGI, 2009). Futuras determinaciones a nivel específico permitirán precisar mejor las preferencias de los grupos hallados.

Este estudio constituye el primer aporte al conocimiento de la comunidad bentónica de tramos pedemontanos del arroyo Achiras-del Gato. La estructura de la biocenosis refleja las condiciones de los hábitats que la albergaban antes de la construcción de la presa, y representa un marco de referencia a partir del cual establecer comparaciones que permitirán evaluar los cambios producidos. Nuestros resultados indican que los muestreos de seguimiento deberían realizarse tanto en el tramo río arriba como en el de aguas abajo de la barrera, en hábitats de corredera o de rabión. Si los muestreos se realizan en períodos hidrológicos de aguas bajas y de aguas altas, un registro semestral del bentos sería representativo del ciclo anual.

Agradecimientos. A la SeCyT (UNRC) por el soporte financiero; a G. Orpella y a P. Ffrench por colaborar en las tareas de campo; a R. Principe y M. Boccolini por contribuir con las determinaciones taxonómicas; a R. Arbeloa por permitirnos el acceso a su campo y a los revisores anónimos que contribuyeron a mejorar este trabajo.

\section{REFERENCIAS BIBLIOGRAFICAS}

Allan, J. D. \& Castillo, M. M. 2007. Stream Ecology. Structure and function of running waters. 2ed. Dordrecht, Springer. 436p.

Armitage, P. D. 2006. Long-term faunal changes in a regulated and an unregulated stream - Cow Green thirty years on. River Research and Applications 22:947-966.

Baptista, D. F.; Dorvillé, L. F. M.; Buss, D. F. \& Nessimian J. L. 2001. Spatial and temporal organization of aquatic insects assemblages in the longitudinal gradient of a tropical river. Revista Brasileira de Biologia 61(2):295-304.

Corigliano, M. C.; Gualdoni, C. M. \& Bosh, B. 2004. Atributos estructurales de ensambles de macroinvertebrados en arroyos de la Pedanía San Bartolomé. Revista de la Universidad Nacional de Río Cuarto 24(1-2):57-69.

Corigliano, M. C.; Gualdoni, C. M.; Oberto, A. M. \& Raffaini, G. B. 1996. Macroinvertebrados acuáticos de Córdoba. In: Di TADA, I. E. \& Bucher, E. H. eds. Biodiversidad de la Provincia de Córdoba: Fauna. Córdoba, Editorial Universidad Nacional de Río Cuarto. p.119-165.

2001. Longitudinal distribution of the mayfly (Ephemeroptera) communities at Chocancharava river basin (Córdoba, Argentina) In: Dominguez, E. ed. Trends in research in Ephemeroptera \& Plecoptera. New York, Kluwer Academic/Plenum Publishers. p.89-96.

Coscarón-Arias, C. L. 2009. Diptera, Simulidae. In: DomingueZ, E. \& FernÁNDEZ, H. R. eds. Macroinvertebrados sudamericanos: Sistemática y biología. Tucumán, Fundación Miguel Lillo. p.365-381. 
Death, R. G. \& Winterbourn, M. J. 1995. Diversity patterns in stream invertebrate communities: the influence of habitat stability. Ecology 76:1446-1460.

Degiovanni, S. 2005. Análisis de problemas geoambientales vinculados a los recursos hídricos en la cuenca del arroyo Achiras-del Gato: Características climáticas, geológico-geomorfológicas y de ocupación territorial. In: Blarasin, M.; Degiovanni, S; Cabrera, A. \& Villegas, M. eds. Aguas superficiales y subterráneas en el sur de Córdoba. Córdoba, Editorial Universidad Nacional de Río Cuarto. p.181-189.

Degiovanni, S.; Blarasin, M. \& Caviglia, L. 2005. Análisis de problemas geoambientales vinculados a los recursos hídricos en la cuenca del arroyo Achiras-del Gato: Análisis de la dinámica composición química e interrelaciones entre el agua superficial y subterránea. In: Blarasin, M.; Degiovanni, S; Cabrera, A. \& Villegas, M. eds. Aguas superficiales y subterráneas en el sur de Córdoba. Córdoba, Editorial Universidad Nacional de Río Cuarto. p.191-202.

Di Rienzo, J. A.; Casanoves, F.; Balzarini, M. G.; Gonzalez, L.; TABLADA, M. \& Robledo, C. W. 2010. InfoStat, software Estadística, v. 2011. Grupo InfoStat, Facultad de Ciencias Agrarias, Universidad Nacional de Córdoba.

DomíngueZ, E. \& FernándeZ, H. 2009. Macroinvertebrados sudamericanos: Sistemática y biología. Tucumán, Fundación Miguel Lillo. 654p.

Dufrene, M. \& Legendre, P. 1997. Species assemblages and indicator species: the need for a flexible asymmetrical approach. Ecological Monographs 67(3):345-366.

Fernández, H. R. \& Dominguez, E. 2001. Guía para la determinación de los artrópodos bentónicos sudamericanos. Tucumán, Universidad Nacional de Tucumán - Facultad de Ciencias Naturales y Instituto Miguel. Lillo. 282p.

Gjerløv, C.; Hildrew, A. G. \& Jones, J. I. 2003. Mobility of stream invertebrates in relation to disturbance and refugia: a test of habitat templet theory. Journal of the North American Benthological Society 22(2):207-223.

Gualdoni, C. M. \& Corigliano, M. C. 1991. El ajuste de un índice biótico para uso regional. Revista de la Universidad Nacional de Río Cuarto 11(1):43-49.

2002. Distribución del bentos y la deriva de macroinvertebrados en tramos fluviales con diferentes condiciones de estrés ambiental. Acta Limnologica Brasiliensia 14(1):1-13.

GuAldoni, C. M. \& OBerto, A. M. 1998. Biological quality assessment in lotic environments of río Carcarañá (Córdoba, Argentina). Verhandlungen Internationale Vereinigung für Theoretische und Angewandte Limnologie 26:1219-1222.

Gualdoni, C. M.; Duarte, C. A. \& Medeot, E. A. 2011. Estado ecológico de dos arroyos serranos del sur de Córdoba, Argentina. Ecología Austral 21:149-162.

Hynes, H. B. N. 1970. The ecology of running waters. Toronto, Toronto University Press. $555 \mathrm{p}$.

LANCASTER, J. 2008. Movement and dispersion of insects of stream channels: What role does flow play? In: LANCASTER, J. \& BRIERS R. A. eds. Aquatic Insects: Challenges to Populations. Wallingford, CABI. p.139-157

MagurRan, A. 1988. Ecological Diversity and its Measurement. Princeton, Princeton University Press. 179p.

Marchese, M. 2009. Annelida, Oligochaeta. In: Domínguez, E. \& FERNÁNDEZ, H. R. eds. Macroinvertebrados sudamericanos: Sistemática y biología. Tucumán, Fundación Miguel Lillo. p.550-565.

Mc Aleece, N. 1997. Biodiversity Profesional Beta 1. London, The Natural History Museum \& The Scottish Association for Marine Science.

Mccune, B. \& Mefford, M. J. 1999. PC-Ord. Multivariate Analysis of Ecological Data. Version 4.0.25. Gleneden Beach, MjM Software.

Miranda, R.; Oscoz, J.; Leunda, P. M.; García-Fresca, C. \& Escala, M. C. 2005. Effects of weir construction on fish population structure in the River Erro (North of Spain). Annales de Limnologie - International Journal of Limnology 41:7-13.
Molineri, C. 2003. Revision of the South American species of Leptohyphes (Ephemeroptera: Leptohyphidae) with a key for the nymphs. Studies on Neotropical Fauna and Environment 38(1):47-70.

2010. Las especies de Leptohyphidae (Ephemeroptera) de las yungas de Argentina y Bolivia: diagnosis, distribución y claves. Revista de la Sociedad Entomológica Argentina 69(3-4):233252.

Ogbeibu, A. E. \& Oribhabor, B. J. 2002. Ecological impact of river impoundment using benthic macro-invertebrates as indicators. Water Research 36:2427-2436.

Paggi, A. C. 2009. Diptera, Chironomidae. In: Domínguez, E. \& FERnÁNDEZ, H. R. eds. Macroinvertebrados sudamericanos: Sistemática y biología. Tucumán, Fundación Miguel Lillo. p.383-409.

Penczak, T.; Kruk, A.; Grzybrowska, M. \& Dukowska, M. 2006. Patterning of impoundment impact on chironomid assemblages and their environment with use of the self-organizing map (SOM). Acta Oecologica 30:312-321.

PofF, N. L. \& HART, D. D. 2002. How dams vary and why it matters for the emerging science of dam removal. BioScience 52:659-668.

PofF, L. N. \& WARD, J. V. 1989. Implications of streamflow variability and predictability for lotic communities structure: a regional analysis of streamflow patterns. Canadian Journal of Fisheries and Aquatic Sciences 45:1805-1818.

1991. Drift responses of benthic invertebrates to experimental streamflow variation in a hidrologically stable stream. Canadian Journal of Fisheries and Aquatic Sciences 48:1926-1936.

Poff, N. L. \& Zimmerman, J. K. H. 2010. Ecological responses to altered flow regimes: a literature review to inform environmental flows science and management. Freshwater Biology 55:194-20.

Poff, N. L.; Oldenn, J. D.; Merritt, D. \& Pepin, D. 2007. Homogenization of regional river dynamics by dams and global biodiversity implications. Proceedings of the National Academcy of Sciences 104:5732-5737.

Poff, N. L.; Allan, J. D.; Bain, M. B.; Karr, J. R.; Prestegaard, K. L.; Richter, B. D.; Sparks, R. E. \& Stromberg, J. C. 1997. The natural flow regime: a paradigm for river conservation and restoration. BioScience 47:769-784.

Principe, R. E.; Gualdoni, C. M.; Oberto, A. M.; Raffaini, G. B. \& Corigliano, M. C. 2010. Spatial-temporal patterns of functional feeding groups in mountain streams of Córdoba, Argentina Ecología Austral 20:257-268.

Principe, R. E.; Raffaini, G. B.; Gualdoni, C. M.; Oberto A M. \& Corigliano, M. C. 2007. Do hydraulic units define macroinvertebrate assemblages in mountain streams of central Argentina? Limnológica 37:323-336.

Rosenberg, M. D. \& Resh, V. H. eds. 1993. Freshwater biomonitoring and benthic macroinvertebrates. New York, Chapman \& Hall. $488 \mathrm{p}$

Smocke, L. A.; Metzler, G. M. \& Gladden, G. E. 1989. Role of debris dams in the structure and function of low-gradient headwater streams. Ecology 70:764-775.

StANFORD, J. A. \& WARD, J. V. 2001. Revisiting the Serial Discontinuity Concept. Regulated River Research \& Management 17:303-210

Strayer, D. L. 2006. Challenges for freshwater invertebrate conservation. Journal of the North American Benthological Society 25:271-287.

Tickner, D.; Armitage, P. D.; Bickerton, M. A. \& Hall, K. A. 2000 Assessing stream quality using information on mesohabita distribution and character. Aquatic Conservation: Marine and Freshwater Ecosystems 10:170-196.

Vinson, M. \& Hawkins, C. 1998. Biodiversity of stream insects: Variation al local, basin, and regional scales. Annual Review of Entomology 43:271-293.

WARD, J. V. 1992. Aquatic Insect Ecology 1. Biology and Habitat New York, John Wiley \& Sons. 384p.

WARD, J. V. \& StANFORD, J. A. 1983. The serial discontinuity concept of lotic ecosystems. In: Fontaine, T. D. \& Bartell, S. M. eds. Dynamics of Lotic Ecosystems. Collingwood, Ann Arbor Sciences. p.29-42.

Recebido em 17 de novembro de 2011. Aceito em 18 de junho de 2012. ISSN 0073-4721

Artigo disponível em: www.scielo.br/isz 